\title{
Classical Casimir interaction in the plane-sphere geometry
}

\author{
Antoine Canaguier-Durand, ${ }^{1, *}$ Gert-Ludwig Ingold, ${ }^{2}$ Marc-Thierry Jaekel, ${ }^{3}$ Astrid Lambrecht, ${ }^{1}$ \\ Paulo A. Maia Neto, ${ }^{4}$ and Serge Reynaud ${ }^{1}$ \\ ${ }^{1}$ Laboratoire Kastler Brossel, ENS, UPMC, Centre National de la Recherche Scientifique, F-75252 Paris, France \\ ${ }^{2}$ Institut für Physik, Universität Augsburg, D-86135 Augsburg, Germany \\ ${ }^{3}$ Laboratoire de Physique Théorique, ENS, UPMC, Centre National de la Recherche Scientifique, F-75231 Paris, France \\ ${ }^{4}$ Instituto de Física, UFRJ, CP 68528, Rio de Janeiro RJ 21941-909, Brazil
}

(Received 6 March 2012; published 1 May 2012)

\begin{abstract}
We study the Casimir interaction in the plane-sphere geometry in the classical limit of high temperatures. In this limit, the finite conductivity of the metallic plates needs to be taken into account. For the Drude model, the classical Casimir interaction is nevertheless found to be independent of the conductivity so that it can be described by a single universal function depending only on the aspect ratio $x=L / R$, where $L$ is the interplate distance and $R$ is the sphere radius. This universal function differs from the one found for perfect reflectors and is in principle amenable to experimental tests. The asymptotic approach of the exact result to the proximity force approximation appears to be well fitted by polynomial expansions in $\ln x$.
\end{abstract}

DOI: 10.1103/PhysRevA.85.052501

\section{INTRODUCTION}

The Casimir effect arises due to the confinement of the quantum fluctuations of electromagnetic fields between reflecting bodies. Its discussion is often focused on the ideal case introduced by H. Casimir [1] of a pair of perfectly reflecting parallel plates at zero temperature. In this case, the energy is given by a universal expression $\mathcal{E}_{0}^{\mathrm{P}}=-\hbar c \pi^{2} A / 720 L^{3}$ depending only on geometrical parameters, the mirrors' separation $L$ and surface $A$, and fundamental constants $\hbar$ and $c$. However, this idealization does not hold for any experiment and reliable descriptions of the Casimir interaction have to account for the optical response of matter [2,3].

In the present article, we want to study another case where the Casimir interaction becomes universal, namely, the high-temperature limit indicated in the following by the subscript HT. This limit has been thoroughly studied for two perfectly reflecting parallel plates [4-6] where the free energy $\mathcal{F}_{\mathrm{HT}}^{\mathrm{P}}=-\zeta(3) k_{\mathrm{B}} T A / 8 \pi L^{2}$ depends only on temperature $T$ and geometrical parameters $L$ and $A$. $\zeta$ is the Riemann function and $\zeta(3) \simeq 1.202$ and the superscript $\mathrm{P}$ refers to perfect mirrors. When the Drude model is used for describing the effect of conduction electrons in metallic mirrors, the free energy $\mathcal{F}_{\mathrm{HT}}^{\mathrm{D}}=\mathcal{F}_{\mathrm{HT}}^{\mathrm{P}} / 2$ is one half of that obtained for perfect mirrors [7-9] (the superscript D refers to the Drude model).

This reduction by a factor of 2 at large temperatures, independent of the parameters of the Drude model (see below), is confirmed by microscopic descriptions of the interaction between two metallic bulks [10-12]. This difference can in principle be tested at room temperature by measuring the force at large distances. Experiments are difficult in this domain because the force decreases with distance. However, experiments have recently been performed at distances up to $7 \mu \mathrm{m}$ where the thermal effect is large. The results have been interpreted by the authors [13] as being in agreement with the Drude model prediction once an electrostatic patch contribution is subtracted [14]. This conclusion stands in contradiction with the results of

*Present address: ISIS_-Université de Strasbourg.
PACS number(s): 31.30.jh, 03.70.+k, 05.70.-a, 78.20.Ci

other Casimir force measurements, performed at smaller distances, up to $0.75 \mu \mathrm{m}$, which were interpreted by their authors as excluding the dissipative Drude model and agreeing with the lossless plasma model [15]. This contradiction remains a matter of debate (see discussions and references in $[16,17]$ ).

It is also worth stressing that most precise experiments are performed in the plane-sphere geometry. The evaluation of the force is often done through the so-called proximity force approximation (PFA) [18], which amounts to averaging the force between parallel plates over the distribution of local interplate distances. This trivial treatment of geometry cannot reproduce the rich relation expected between Casimir effect and geometry [17,19-21]. Theoretical treatments going beyond the PFA have recently been proposed, in particular through a multipolar expansion well adapted to the plane-sphere geometry. In the following, we will use results known for perfect or metallic mirrors coupled to electromagnetic fields $[22,23]$.

The aim of the present article is to investigate the classical Casimir interaction for the geometry of a plane and a sphere with an arbitrary aspect ratio. We will focus our attention on the models of perfect mirrors and dissipative mirrors and use scattering methods discussed in [23]. The results will turn out to be given by universal functions of the aspect ratio, for dissipative as well as perfect mirrors, with the two functions differing from each other. The limit of high temperatures will allow us to reach a much better numerical accuracy than in previous works [23]. This improvement in the accuracy will be used to sharpen the discussion of the asymptotic approach of the exact result to the PFA. This asymptotic behavior will appear to be well fitted by polynomial expansions of the logarithm of the aspect ratio.

\section{THE SCATTERING FORMULA AT THE CLASSICAL LIMIT}

We start from the Matsubara formula giving the Casimir free energy $\mathcal{F}$ between a plane and a sphere [23]:

$$
\begin{aligned}
& \mathcal{F}=k_{\mathrm{B}} T \sum_{n}^{\prime} \ln \operatorname{det} \mathcal{D}\left(i \xi_{n}\right), \\
& \mathcal{D}=\mathcal{I}-\mathcal{R}_{2} e^{-\mathcal{K L}} \mathcal{R}_{1} e^{-\mathcal{K L}} .
\end{aligned}
$$


Here $\xi_{n}=2 \pi n k_{\mathrm{B}} T / \hbar$ are the Matsubara frequencies and the prime indicates a sum over integers $n=0 \ldots \infty$ with a factor $\frac{1}{2}$ for $n=0 ; \mathcal{R}_{1}$ and $\mathcal{R}_{2}$ are the reflection operators on the plane and the sphere, respectively, discussed below in terms of Fresnel reflection amplitudes and Mie scattering amplitudes; $e^{-\mathcal{K} \mathcal{L}}$ describes the one-way propagation on the distance $\mathcal{L}=$ $L+R$ between the center of the sphere and the plane, with $R$ being the radius of the sphere and $L$ being the minimal distance between the plane and the sphere; $\mathcal{K}=\sqrt{k^{2}+\xi^{2} / c^{2}}$ is the longitudinal wave vector after a Wick rotation from real to imaginary frequencies, with $k$ being the modulus of the transverse wave vector.

The nonzero Matsubara frequencies $\xi_{n \neq 0} \geqslant 2 \pi k_{\mathrm{B}} T / \hbar$ increase with temperature and their contributions to the sum Eq. (1) become exponentially small because of the propagation factor $e^{-\mathcal{K} \mathcal{L}}$ with $\mathcal{K} \geqslant \xi / c \geqslant 2 \pi k_{\mathrm{B}} T / \hbar c$. At the hightemperature limit $k_{\mathrm{B}} T \gg \hbar c / \mathcal{L}$, the free energy is dominated by the first term $n=0$ in the sum Eq. (1) and, therefore, it is proportional to temperature:

$$
\begin{aligned}
& \mathcal{F}_{\mathrm{HT}}=-k_{\mathrm{B}} T \Phi, \quad \Phi=-\frac{1}{2} \ln \operatorname{det} \mathcal{D}(0), \\
& \mathcal{D}(0)=\mathcal{I}-\mathcal{R}_{S}(0) e^{-k \mathcal{L}_{\mathcal{R}}} \mathcal{R}_{P}(0) e^{-k \mathcal{L}} .
\end{aligned}
$$

$\Phi$ is a dimensionless function of the parameters which does not depend on temperature. Hence the entropy $\mathcal{S}$ is independent of temperature and the energy $\mathcal{E}$ vanishes:

$$
\begin{aligned}
& \mathcal{S}_{\mathrm{HT}}=-\partial_{T} \mathcal{F}=k_{\mathrm{B}} \Phi, \\
& \mathcal{E}_{\mathrm{HT}}=\mathcal{F}_{\mathrm{HT}}+T \mathcal{S}_{\mathrm{HT}}=0 .
\end{aligned}
$$

This implies that the classical limit of the Casimir interaction has a purely entropic origin [24,25].

The Drude model is the simplest description of a dissipative mirror. It corresponds to a dielectric function $\varepsilon[i \xi]=1+\sigma / \xi$ at imaginary frequencies $\omega=i \xi$, without a magnetic response $(\mu=1) . \sigma[i \xi]=\omega_{\mathrm{p}}^{2} /(\gamma+\xi)$ measures the conductivity associated with conduction electrons (the conductivity in SI units is $\epsilon_{0} \sigma$ ). The Drude model is characterized by two dimensional parameters, the squared plasma frequency $\omega_{\mathrm{p}}^{2}$, proportional to the density of the conduction electrons, and their relaxation rate $\gamma$. It meets the important property of ordinary metals to have a finite static conductivity $\sigma_{0}=\omega_{\mathrm{p}}^{2} / \gamma$. The lossless plasma model $\gamma \rightarrow 0$ corresponds to an infinite value for $\sigma_{0}$, in contradiction with tabulated optical data at low frequencies [26,27].

The Fresnel reflection amplitudes $r_{\mathrm{TE}}$ and $r_{\mathrm{TM}}$ for plane waves with polarization TE or TM are well known from studies of the plane-plane geometry [7-9]. They are $r_{\mathrm{TE}}^{\mathrm{P}} \rightarrow-1$ and $r_{\mathrm{TM}}^{\mathrm{P}} \rightarrow 1$ for perfect mirrors whereas they go to $r_{\mathrm{TE}}^{\mathrm{D}} \rightarrow 0$ and $r_{\mathrm{TM}}^{\mathrm{D}} \rightarrow 1$ for the Drude model at low frequencies. For the lossless plasma model, they are found to interpolate between perfect and Drude models, a property found also below for the Mie amplitudes. The disappearance of the TE reflection amplitude is responsible for the ratio 2 between the classical limits of the free energies obtained from perfect and Drude models in the plane-plane geometry.

A similar discrepancy arises also for the Mie amplitudes $a_{\ell}$ and $b_{\ell}$ which describe the scattering on the sphere [24]. For perfect mirrors and low frequencies, we get $a_{\ell}^{\mathrm{P}} \simeq \frac{(-1)^{\ell}(\ell+1) \tilde{\xi}^{(2 \ell+1)}}{\ell(2 \ell+1) ! !(2 \ell-1) ! !}$ and $b_{\ell}^{\mathrm{P}} \simeq-\frac{a_{\ell}^{\mathrm{P} \ell}}{\ell+1}$. The electric and magnetic multipoles $a_{\ell}$ and $b_{\ell}$ progressively decrease with increasing multipole index $\ell$, but they have the same power law in the reduced frequency $\tilde{\xi}=\xi R / c$ for a given $\ell$. The situation is different for the Drude model where $a_{\ell}^{\mathrm{D}} \simeq a_{\ell}^{\mathrm{P}}$ and $b_{\ell}^{\mathrm{D}} \simeq \frac{b_{\ell}^{\mathrm{P}} \tilde{\sigma}_{0} \tilde{\xi}}{(2 \ell+3)(2 \ell+1)}\left(\tilde{\sigma}_{0}=\sigma_{0} R / c\right.$ is the reduced frequency associated with the static conductivity). Incidentally, the results for the lossless plasma model are found to interpolate between perfect and Drude models: $b_{\ell}$ has the same form as $b_{\ell}^{\mathrm{P}}\left(b_{\ell}^{\mathrm{D}}\right)$ for large spheres (small spheres). The crossover between these two regimes is determined by the parameter $\tilde{\omega}_{\mathrm{p}}$ being, respectively, larger or smaller than 1 ( $\tilde{\omega}_{\mathrm{p}}=\omega_{\mathrm{p}} R / c$ is the reduced plasma frequency).

The function $\Phi^{\mathrm{P}}$ is a universal expression of the aspect ratio for perfect mirrors, as there exist no other parameters. The discussion of the preceding paragraph entails that it is also the case for the function $\Phi^{\mathrm{D}}$ calculated for Drude mirrors [28], although the latter is associated with dimensional parameters like $\sigma_{0}$. As a matter of fact, $b_{\ell}$ is negligible with respect to $a_{\ell}$ for any given value of $\ell$, and it follows that the classical high-temperature limit is the same as if $b_{\ell}^{\mathrm{D}}$ were simply zero. In spite of the fact that $\Phi^{\mathrm{D}}$ and $\Phi^{\mathrm{P}}$ are universal functions of the aspect ratio, these two functions differ. As another important consequence of this discussion is that the lossless plasma model result does not obey the universality property met by perfect as well as Drude mirrors. For this reason, we will not discuss this model any longer in this article.

\section{NUMERICAL EVALUATIONS AND RESULTS}

In the remainder of this article, we present the results of the numerical evaluation of the functions $\Phi^{\mathrm{D}, \mathrm{P}}$ which determine the classical Casimir free energy between a plane and a sphere, for Drude and perfect mirrors, respectively. We write these functions through a comparison with the often used PFA approximation (the superscript D,P means D or P):

$$
\begin{aligned}
\Phi^{\mathrm{D}, \mathrm{P}}(x) & =\frac{C^{\mathrm{D}, \mathrm{P}}}{x} \varrho^{\mathrm{D}, \mathrm{P}}(x), \quad x \equiv \frac{L}{R}, \\
C^{\mathrm{D}} & \equiv \frac{\zeta(3)}{8}, \quad C^{\mathrm{P}} \equiv \frac{\zeta(3)}{4} .
\end{aligned}
$$



FIG. 1. (Color online) Classical (high-temperature) Casimir interaction between plane and spherical mirrors, represented by the ratio $\varrho^{\mathrm{D}, \mathrm{P}}$ of the exact result to the PFA result vs the aspect ratio $x=L / R$; the full red line corresponds to Drude mirrors and the dashed blue line corresponds to perfect mirrors. 
The first factors in the expressions of $\Phi^{\mathrm{D}, \mathrm{P}}$ are the PFA results while the second ones, $\varrho^{\mathrm{D}, \mathrm{P}}$, represent deviations from PFA. The factors $\varrho^{\mathrm{D}, \mathrm{P}}$ go to one for large spheres $x \rightarrow 0$, which confirms the validity of PFA as an asymptotic approximation. The fact that $\Phi^{\mathrm{D}, \mathrm{P}}$ and $\varrho^{\mathrm{D}, \mathrm{P}}$ depend on a single geometrical parameter, the aspect ratio $x$, corresponds to the universality discussed above.

The explicit numerical evaluation proceeds as in [23]. Due to the relatively simple form of the matrices in the low-frequency limit, the numerics can be pushed farther than for the general case. Precisely, the computation can be pushed to a larger maximum value $\ell_{\max }$ for the multipole index $\ell$, and it follows that the numerical results are now more accurate for small values of the aspect ratio $x$. The results of the numerical evaluations shown on Fig. 1 have been calculated with $\ell_{\max }$ up to 5000 at our minimal value for the aspect ratio $10^{-3}$. The numerical accuracy $\delta \varrho$ is estimated to be of the order of $10^{-4}$ at $x=10^{-3}$ and of $10^{-8}$ at $x=2 \times 10^{-3}$.

Though both of them are universal, the functions $\varrho^{\mathrm{D}}(x)$ and $\varrho^{\mathrm{P}}(x)$ drawn in Fig. 1 do not coincide. In other words, the ratio $\Phi^{\mathrm{P}}(x) / \Phi^{\mathrm{D}}(x)=2 \varrho^{\mathrm{P}}(x) / \varrho^{\mathrm{D}}(x)$ between Casimir free energies
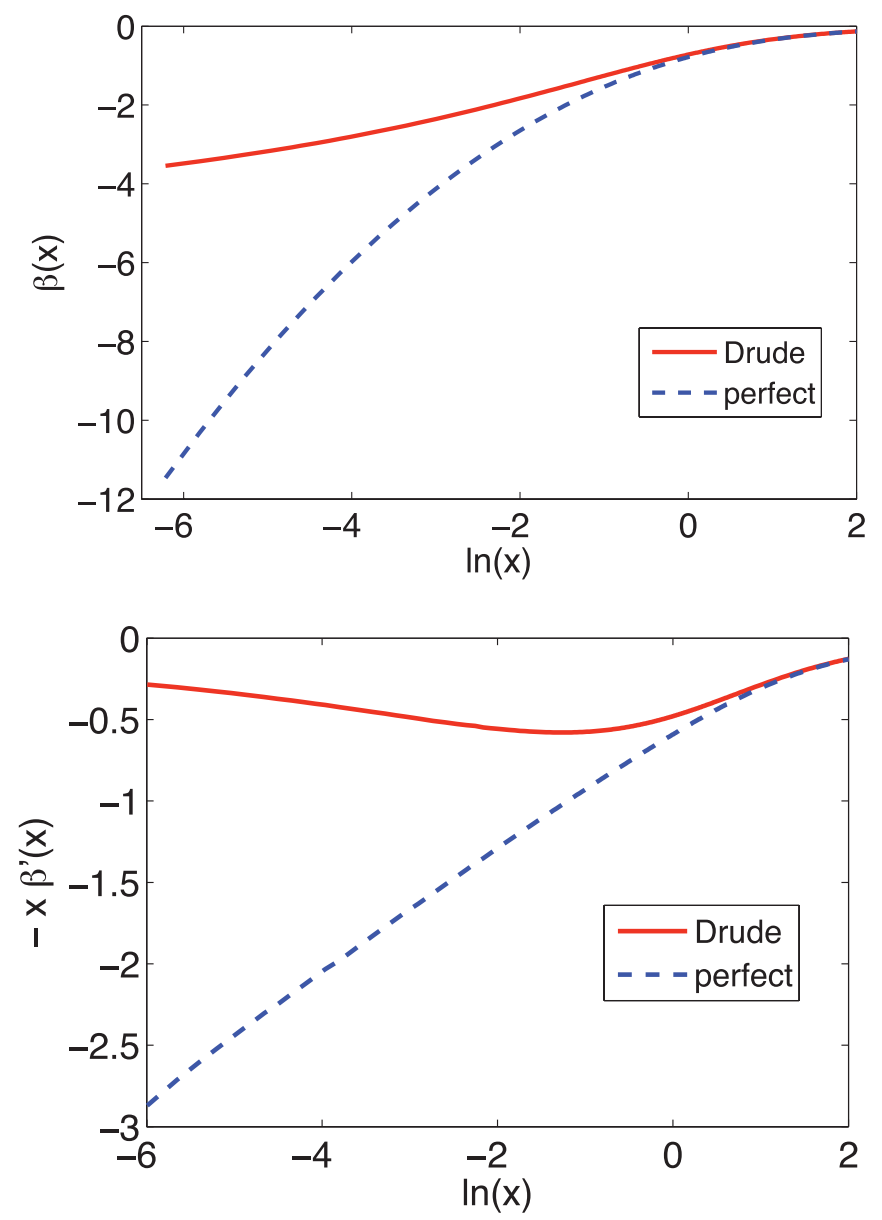

FIG. 2. (Color online) Deviation from PFA of the classical Casimir interaction between plane and spherical mirrors, described by the additive correction $\beta^{\mathrm{D}, \mathrm{P}}(x)$ vs $\ln x$ (upper plot) and the logarithmic derivatives $-\frac{\mathrm{d} \beta^{\mathrm{D}, \mathrm{P}}}{\mathrm{d} \ln x}=-x \frac{\mathrm{d} \beta^{\mathrm{D}, \mathrm{P}}}{\mathrm{d} x}$ (lower plot); the full red lines correspond to Drude mirrors and the dashed blue lines correspond to perfect mirrors. calculated for perfect and Drude mirrors is itself a function of $x$. This ratio reaches the value 2 at the PFA limit $(x \rightarrow 0)$, as known from the studies on plane-plane geometry [7], and the value $3 / 2$ at large distances $(x \rightarrow \infty)$ [23].

\section{ASYMPTOTIC APPROACH TO PFA}

We finally discuss the asymptotic approach to PFA for small values of $x$. To this aim, we introduce another representation of the deviation from PFA:

$$
\varrho^{\mathrm{D}, \mathrm{P}}(x) \equiv 1+x \beta^{\mathrm{D}, \mathrm{P}}(x) .
$$

$\beta^{\mathrm{D}, \mathrm{P}}(x)$ is the slope of the line which joins the points $(0,1)$ to $\left(x, \varrho^{\mathrm{D}, \mathrm{P}}(x)\right)$ on the plots of Fig. 1. It may also be thought of as an additive correction to PFA in Eq. (4):

$$
\Phi^{\mathrm{D}, \mathrm{P}}(x)=C^{\mathrm{D}, \mathrm{P}}\left(\frac{1}{x}+\beta^{\mathrm{D}, \mathrm{P}}(x)\right) .
$$

The numerical accuracy $\delta \varrho$ discussed above is now translated into a numerical accuracy $\delta \beta=\delta \varrho / x$ for $\beta$. This accuracy $\delta \beta$ is estimated to be of the order of $10^{-1}$ at $x=10^{-3}$ and of $5 \times 10^{-6}$ at $x=2 \times 10^{-3}$. The following discussions of the asymptotic approach to PFA are possible only thanks to this good accuracy.

The quantities $\beta^{\mathrm{D}, \mathrm{P}}$ are shown as the upper plot in Fig. 2 for Drude and perfect mirrors, as functions of $\ln x$. The lower
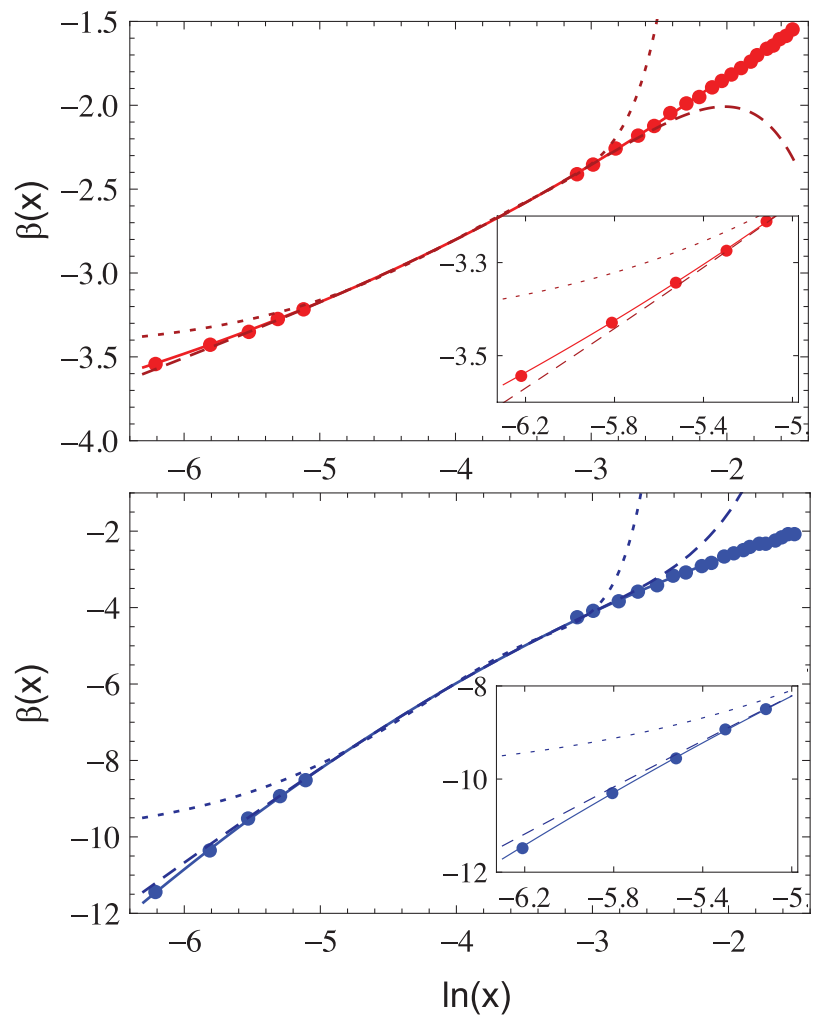

FIG. 3. (Color online) Best fits of the numerical results with the trial functions Eq. (8) for the Drude (red lines, upper plot) and perfect mirrors (blue lines, lower plot); the full, dotted, and dashed lines correspond, respectively, to $\beta_{l}, \beta_{x}$, and $\beta_{m}$; the full circles correspond to numerical points not used for the fits; the insets zoom on the smallest values of $x$. 
plot in Fig. 2 represents the quantities $-\frac{\mathrm{d} \beta^{\mathrm{D}, \mathrm{P}}}{\mathrm{d} \ln x}$ which appear in the Casimir force:

$$
F^{\mathrm{D}, \mathrm{P}}(x)=-\frac{C^{\mathrm{D}, \mathrm{P}} k_{\mathrm{B}} T}{L}\left(\frac{1}{x}-\frac{\mathrm{d} \beta^{\mathrm{D}, \mathrm{P}}(x)}{\mathrm{d} \ln x}\right) .
$$

The plots in Fig. 2 suggest that the asymptotic approach to PFA is well described for small values of $x$ by a polynomial expansion in the variable $\ln x$. In order to check this idea, we have performed best fits of the numerical results with the following trial functions, defined with the same number of parameters:

$$
\begin{aligned}
& \beta_{l}(x)=a_{0}+a_{1} \ln x+a_{2} \ln ^{2} x+a_{3} \ln ^{3} x, \\
& \beta_{x}(x)=b_{0}+b_{1} x+b_{2} x^{2}+b_{3} x^{3} \\
& \beta_{m}(x)=c_{0}+c_{1} \ln x+c_{2} x+c_{3} x^{2} .
\end{aligned}
$$

The first one, $\beta_{l}$, is a polynomial form in the variable $\ln x$ as suggested by Fig. 2 (see also [29]). The second one, $\beta_{x}$, is a polynomial form in the variable $x$, which was sufficient to represent functions $\rho$ obtained from older calculations with a lesser accuracy $[22,30]$. It corresponds to the free energy being a Laurent series in the variable $x$. The third one, $\beta_{m}$, is a mixed form which results, in the classical limit (high temperatures), from the assumption [31] that the force (not the free energy) may be written as a Laurent series in $x$.
In each case, the best-fit functions are defined from the numerical results obtained in the window $\ln x \in[-5,-3]$. Their comparison in Fig. 3 with the numerical values obtained in a broader window shows that $\beta_{1}$ is a better representation of the numerical results than $\beta_{m}$, itself better than $\beta_{x}$. In particular, the insets in Fig. 3 show the deviations of the best fits of $\beta_{x}$ and $\beta_{m}$ from the exact results at the smallest values of the aspect ratio $\ln x \in[-6.2,-5]$. The assumptions that the free energy or force is a Laurent series in $x$ cannot be considered as generally valid.

The difference between the cases of Drude and perfect mirrors is in principle amenable to experimental tests. Direct comparison with exact calculations would require measurements at large distances (high-temperature limit). This challenge could be met with experimental parameters not so far from those in the experiment [13].

\section{ACKNOWLEDGMENTS}

The authors thank the European Science Foundation Research Networking Program CASIMIR (www.casimirnetwork.com) for providing excellent possibilities for discussions and exchange. Financial support from international programs Capes-Cofecub and Procope is gratefully acknowledged.
[1] H. B. G. Casimir, Proc. K. Ned. Akad. Wet. 51, 793 (1948).

[2] E. M. Lifshitz, Sov. Phys. JETP 2, 73 (1956).

[3] M.-T. Jaekel and S. Reynaud, J. Physique I 1, 1395 (1991).

[4] J. Mehra, Physica 37, 145 (1967).

[5] L. S. Brown and G. J. Maclay, Phys. Rev. 184, 1272 (1969).

[6] J. Schwinger, L. L. de Raad, and K. A. Milton, Ann. Phys. (NY) 115, 1 (1978).

[7] M. Boström and B. E. Sernelius, Phys. Rev. Lett. 84, 4757 (2000).

[8] I. Brevik, S. E. Ellingsen, and K. A. Milton, New J. Phys. 8, 236 (2006).

[9] G.-L. Ingold, A. Lambrecht, and S. Reynaud, Phys. Rev. E 80, 041113 (2009).

[10] B. Jancovici and L. Šamaj, Europhys. Lett. 72, 35 (2005).

[11] P. R. Buenzli and P. A. Martin, Europhys. Lett. 72, 42 (2005).

[12] G. Bimonte, Phys. Rev. A 79, 042107 (2009).

[13] A. O. Sushkov, W. J. Kim, D. A. R. Dalvit, and S. K. Lamoreaux, Nat. Phys. 7, 230 (2011).

[14] R. O. Behunin, F. Intravaia, D. A. R. Dalvit, P. A. Maia Neto, and S. Reynaud, Phys. Rev. A 85, 012504 (2012).

[15] R. S. Decca, D. López, E. Fischbach, G. L. Klimchitskaya, D. E. Krause, and V. M. Mostepanenko, Ann. Phys. (NY) 318, 37 (2005); Phys. Rev. D 75, 077101 (2007).

[16] G. L. Klimchitskaya, U. Mohideen, and V. M. Mostepanenko, Rev. Mod. Phys. 81, 1827 (2009).

[17] A. Lambrecht, A. Canaguier-Durand, R. Guérout, and S. Reynaud, in Casimir Physics, edited by D. A. R. Dalvit et al., Lecture Notes in Physics, Vol. 834 (Springer-Verlag, New York, 2011), p. 97.
[18] B. V. Derjaguin, I. I. Abrikosova, and E. M. Lifshitz, Q. Rev. Chem. Soc. 10, 295 (1968).

[19] R. Balian and B. Duplantier, Ann. Phys. (NY) 104, 300 (1977); 112, 165 (1978).

[20] A. Weber and H. Gies, Phys. Rev. D 82, 125019 (2010).

[21] S. J. Rahi, T. Emig, and R. L. Jaffe, in Casimir Physics, edited by D. A. R. Dalvit et al., Lecture Notes in Physics, Vol. 834 (Springer-Verlag, New York, 2011), p. 129.

[22] P. A. Maia Neto, A. Lambrecht, and S. Reynaud, Phys. Rev. A 78, 012115 (2008); A. Canaguier-Durand, P. A. Maia Neto, I. Cavero-Pelaez, A. Lambrecht, and S. Reynaud, Phys. Rev. Lett. 102, 230404 (2009).

[23] A. Canaguier-Durand, P. A. Maia Neto, A. Lambrecht, and S. Reynaud, Phys. Rev. Lett. 104, 040403 (2010); Phys. Rev. A 82, 012511 (2010).

[24] J. Feinberg, A. Mann, and M. Revzen, Ann. Phys. (NY) 288, 103 (2001).

[25] L. Spruch, Phys. Rev. A 66, 022103 (2002).

[26] A. Lambrecht and S. Reynaud, Eur. Phys. J. D 8, 309 (2000)

[27] V. B. Svetovoy, P. J. van Zwol, G. Palasantzas, and J. T. M. De Hosson, Phys. Rev. B 77, 035439 (2008).

[28] R. Zandi, T. Emig, and U. Mohideen, Phys. Rev. B 81, 195423 (2010).

[29] M. Bordag and V. Nikolaev, Phys. Rev. D 81, 065011 (2010).

[30] T. Emig, J. Stat. Mech.: Theory Exp. (2008) P04007.

[31] G. Bimonte, T. Emig, R. L. Jaffe, and M. Kardar, Europhys. Lett. 97, 50001 (2012). 